\title{
A Selective reliable communication to reduce broadcasting for cluster based VANET
}

\author{
R. Shiddharthy ${ }^{a}$, Dr R. Gunavathi ${ }^{b}$ \\ ${ }^{a}$ Research Scholar, Department of Computer Applications, Sree Saraswathi Thyagaraja College, Pollachi, India \\ ${ }^{\mathrm{b}}$ Associate Professor, Department of Computer Applications, Sree Saraswathi Thyagaraja College, Pollachi, India \\ ashiddharthy@gmail.com, bgunaganesh2001@gmail.com
}

Article History: Received: 10 November 2020; Revised 12 January 2021 Accepted: 27 January 2021; Published online: 5 April 2021

\begin{abstract}
Vehicular Ad hoc Network (VANET) is one of the subset of Mobile Ad hoc Network (MANET) and it is a selforganised system with a group of vehicles, which are capable of short-range communication using On Board Unit (OBU). This unit is comprised with the vehicles that are possible to communicate with the nearby vehicles. VANETs rely on heavy broadcast transmission due to sharing data (messages) between the nearby vehicles about the traffic, collision and so on. This redundant information spoils the nature of VANET that affects the inter-vehicular communication, rebroadcasting and information on collision. This message transmission increases largely as the number of vehicles increases. This problem is typically named as broadcast storm and it is relatively reduced through the proposed Selective Reliable Communication (SRC) Protocol. Through a reliable communication, packets are retransmitted to reduce a number of transmission in the network within the acceptable level of QoS. The proposed SRC protocol automatically detect the vehicle clusters as "Zone of Interest". Generally, the proposed protocol forwards the packets to the cluster-heads and the cluster-head forwards the packets to the cluster-members. The proposed protocol outperforms than the existing protocols in terms of Throughput, Packet Delivery Ratio (PDR) and Average delay.
\end{abstract}

Keywords: VANET; MANET; QoS; Reliable communication; cluster communication

\section{Introduction}

VANETs are emerging from MANET where the network is preferably design for the Intelligent Transport System (ITS) which provides an inter-vehiclular short range communication for the common support of safety applications. As rapid growth in transportation system, the traffic and other vehicle related issues were increased in the past decades. Due to this, road accidents and traffic congestions are noticed huge in the recent years. World Health Organisation (WHO) reports that the over 1 million deaths are causing every year due to vehicle accidents [1-4]. Therefore the need of road safety, traffic management information and other relevant information are to be shared with the vehicles using a defined network called VANET. Generally, VANET provide vehicle-to-vehicle (V2V) communication and Vehicle-to-infrastructure (V2I) communication to share information between vehicles [3]. VANETs is an ITS with the composed of interconnected vehicles and Road Side Units (RSU).

$\mathrm{V} 2 \mathrm{~V}$ communication is accomplished using some hardware and software equipments that are specially made for VANETS [5-7]. They are, OBU with Network Interface Cards (NIC), which can connect to IEEE 802.11p, WiMAX, Long Term Evolution (LTE), Global Navigation Satellite System (GNSS) receiver and so on. Vehicles are connected together to form a cluster that can communicate easily. However, communication between different clusters may disrupted due to some of the network topology changes, variable speed in between vehicles and in disconnected scenarios [8].

Moreover, the message transmission and duplication of messages are increased heavily and detains the performance of the network. The data transmission is carried out to discover neighboring vehicles and to transfer traffic-related information to the nearby vehicles through context-aware applications. Even though the broadcasting is achieved, it may lead to frequent contention and collision because of redundant transmission between the vehicles in a high density network [9-11]. This problem is generally referred as broadcast storm problem and it affects inter-vehicle communications with increased contention and collision through rebroadcasting of a message between the vehicles [10]. 


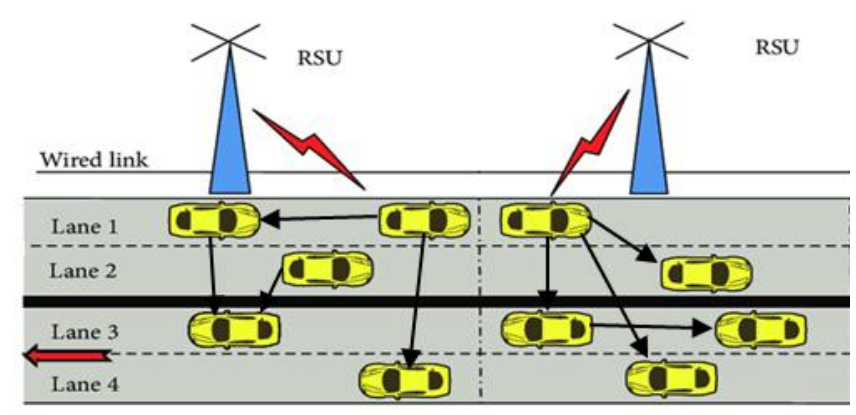

Fig. 1. VANET Architecture.

The Figure 1 shows the basic architecture of VANET. Here the vehicles receives the messages from the RSU and forwards the data to the nearby vehicles. RSUs are associated with the wired link and it forwards the data through wireless mode.

In a MANET environment, solutions are proposed to alleviate the broadcast storm affect in VANET environment. Some recent research works focused on analysing VANET as a well-connected network that provides a high traffic density. As vehicles in close area, sense the traffic situation of the same situation broadcast the message to the other vehicles, which leads to an excessive message redundancy. on the other side low vehicular density with RSU and low traffic results in low and poor network connectivity and intermittent. The design of reliable and efficient routing protocol is a challenge. Suppose a hybrid solution is an effective way to propose an alternate routing protocol to improve the V2V communication [12].

This paper presents a selective reliable communication protocol to provide a better cluster-based broadcasting technique. The paper is organised in the subsequent sections: Section II provides information about Literature review of the past works in the same area. Section III presents the problem statement and Section IV tells about the proposed SRC protocol. Section V presents simulation results and Section VI concludes the paper with conclusion and future work.

\section{Related work}

This section details the previous works of VANET to avoid the broadcast storm in cluster-based approaches.

Benrhaiem et al. propose multi-Hop Reliable Broadcasting (M-HRB), for wide range of VANET applications for the urban area [13]. The protocol is proposed based on local state information where the streets are divided into multiple cells. These multiple cells are formed together to form grid-like zones. A Proactive local state processing is proposed to exploit features of periodic beacons. Thus estimates the neighbour's quality and adequate forwarders are identified and achieve desirable reliability in each hop in multi-hop broadcasting. Additionally the consumption bandwidth is minimised and it improves the lifetime of the network. M-HRB attains better performance than the existing schemes in terms of reliability and bandwidth consumption. Even though it achieves better performance in terms of reliability and selection of forwarders is not reliable in MANET where the selection of forwarders is to be maintained and selected for each transmission that setbacks the performance in a network with a large number of vehicles.

Selvi \& Ramakrishnan presents an efficient message prioritization technique with the scheduled partition for transferring emergency message in VANET [14]. The work focused in prioritizing the messages before beginning the transmission reduces the rebroadcasting of same messages to the same nodes. Therefore, the first priority is focused in prioritizing the transmission in VANET. To prioritize, data identification is focused in partitioning the data as normal data or emergency related data. As emergency data is high prior to reach the nodes compare to the normal data. Thus, the emergency data transmission is identified as high priority and transfer it to the nodes. Second, the emergency transmission and normal data transmission is processed using two techniques named, i) based on Similarity metrics of the SMTP and ii) based on the adaptive scheduled partitioning technique. The SMTP flows with normal data and adaptive scheduled technique follows for emergency message transmission. This schemes attains better results in data transmission yet, choosing the transmission technique and detecting the message type is a tedious process where the number of messages are huge and transmission range is high.

Pramuanyat et al., proposed a location based reliable broadcasting for VANET [15]. ITS in VANET enables a huge number of safety applications where these applications requires speed and safety transmission, reliability and restricted area dissemination for transferring high priority information to the right vehicles. The ability of location awareness is identified mostly using Global Positioning Systems (GPS), which gives inaccuracy in closed area. Therefore, the proposed work focused in reliable broadcasting protocol relies on Distributed Energy Conservation 
Energy (DECA). DECA provides a better location services to identify the nodes in VANET. Thus, the accuracy of data transmission is improvised.

Oliveira et al., proposed a reliable data transmission protocol to transfer traffic safety information in VANET [16]. One of the major challenge in VANET is to design an adaptive broadcast protocol to detect the broadcast storm. This work proposed a novel Adaptive data dissemination Protocol (Addp) to handle the broadcast storm using periodical and dynamical adjustment for beacon periodicity and reducing the beacons and messages in the network. The effectiveness of the proposed protocol is evaluated and attains better performance than the other existing protocols. Even though the proposed work achieves better performance than the existing work in throughput for urban areas, the same protocol detains its level to a minimal throughput for large metro areas.

Ramalingam \& Thangarajan focused in clustering based on obtained weight value and disseminating the emergency message through Selective Reliable Broadcasting (SRB) [17]. Generally, vehicles are manufactured with an onboard unit to communicate with other vehicles for overall driving experience and security. V2V and V2I communication provides vehicles with route and traffic information but the dynamic topology of VANET maintains frequent disconnection however, the proposed work guarantees efficient cluster formation and maintenance based on the proposed weighted cluster algorithm. Emergency message is disseminated based on the proposed SRB protocol. The proposed protocol achieves a better performance than the other existing protocols in terms of throughput and data transmission.

Abbasi et al., proposed a fast and reliable nultihop routing protocol called Intelligent Forwarding Protocol (IFP) in VANET for disseminating safety messages (overall safety messages) between the vehicles [18]s. In an dynamic environment many protocols are proposed to share safety messaged among vehicles. Most of the proposed work performs adequately under the limited and minimal traffic conditions. The proposed protocol exploits handshake-less communication with ACK decoupling for efficient collision resolution. IFP is theoretically modelled using simulation and real-world experiment. The message propogation delay is reduced and thus improves the Packet Delivery Ratio (PDR) of the proposed protocol.

Sattar et al., proposed reliability and energy-efficiency on safety message broadcast in VANET [19]. The model focused in reliability of flooding as an underlying data dissemination protocol to deliver time-critical safety message. The end-to-end reliability is provided through the network layer and it results insights about the flooding mechanism. Maintaining the threshold value after a certain rounds of message improves the PDR rate that results in improving the lifetime of the network. The energy-efficient protocols is a key requirement in the upcoming Internet of Vehicles (IoV) and the proposed protocol validates the improvements through simulation results with the existing schemes.

The above existing works shows that the broadcast storm is an important issue in VANET to resolve to obtain better network model. The proposed work SRC focused in minimising the broadcast storm through retransmitting the messages to the network with the acceptable level of QoS.

\section{Problem Statement}

The proposed SRC protocol focused in reducing the broadcast storm. Some common problems due to broadcast storm in VANET is,

- $\quad$ It is the accumulation of broadcast and multicast traffic.

- $\quad$ The switches repeatedly rebroadcast the broadcast messages and flooding in the network.

- $\quad$ Layer 2 header does not support a time to live (TTL).

- Whenever a frame is sent to a looped topology then it loops forever.

The proposed work focused in minimizing the number of rebroadcast through limiting the number of packet transmission. Through implementing Connected Set of Vehicles (CSV) and Eliminated Set of Vehicles (ESV) in a GPS equipped vehicles to broadcast the intensive beacon message between the nearby vehicles. The proposed SRC details in the next section.

\section{Proposed SRC Protocol}

The proposed work focused in SRC protocol to limits the broadcast storm. The protocol maintains the vehicles as two distinct sets named CSV and ESV. The block diagram of the SRC protocol is shows in Fig. 2.

Before the broadcasting begins, the reliability of the vehicle is checked. Based on the reliability the vehicles are classified into CSV and ESV. Through CSV the broadcasting of message transmission will successfully transmitted. Whenever the reliability of vehicles ends with ESV set, then the broadcasting is eliminated from the vehicle and transmission is stopped. 


\section{Connected Set of Vehicles}

The CSV is identified based on the reliability of the vehicle. Whereas, the speed of the vehicle and timestamp is normally generated with the basic packets structure. Based on the timestamp and the basic threshold value the vehicle will be considered as CSV or not. Whenever the timestamp attains greater value then the vehicle remains in the CSV. On

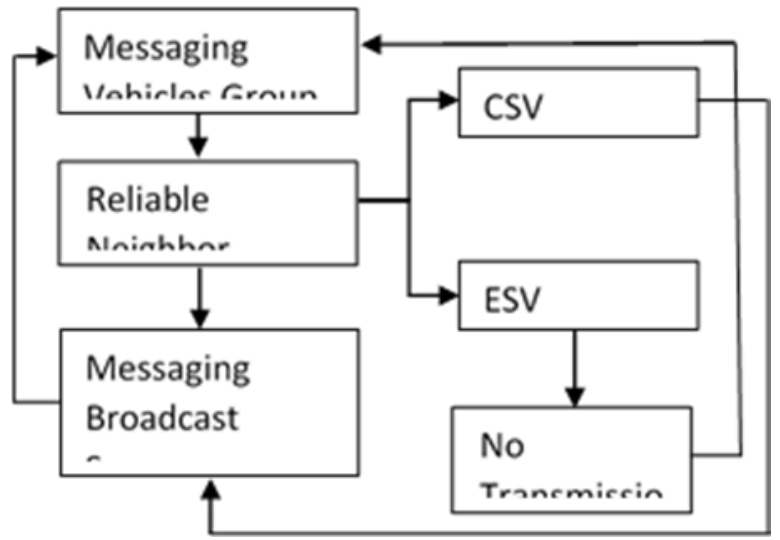

Figure 2. Block diagram of SRC Protocol

successful verification of reliability then the message transmission will be processed.

\section{Eliminated Set of Vehicles}

The ESV set generally joins after the CSV check. Whenever the vehicles fails to join into CSV then the vehicle will be automatically joins to ESV. For every messages the vehicle will be recheck its threshold value and timestamp value to group the vehicle into CSV or ESV.

Additionally the vehicles in ESV checks with distance of the vehicles and the basic threshold value of distance. Whenever the vehicle attains a less distance than the threshold distance then ofcourse those vehicles are reconsidered to join as CSV else the vehicle will remains in ESV.

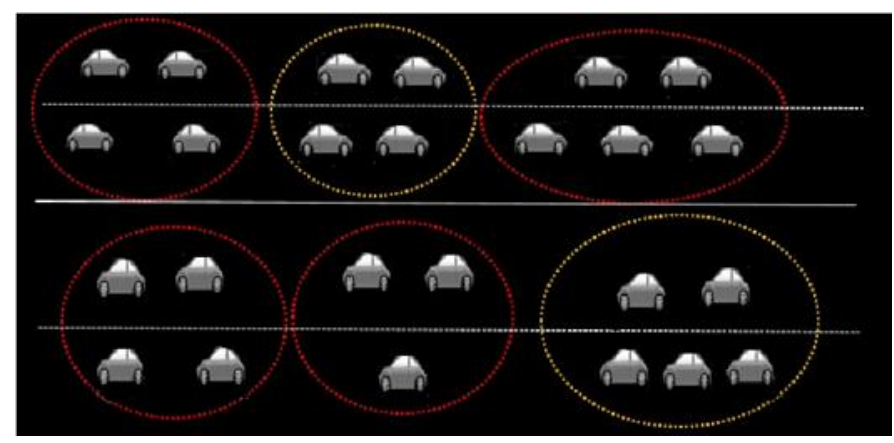

Figure 3. Proposed CSV and ESV

The Fig. 3. shows the proposed SRC protocol with CSV and ESV. the red dotted circle is a CSV and yellow dotted is mentioned as ESV. Both the sets may vary due to time, speed and other common parameters. Therefore, the data transmission will be processed before each message transmission.

Generally, the cluster detection is formulated based on the basic architecture of the VANET. Here the SRC adds cluster detection method to form a better cluster under the three different cases:

- Case - 1: the defined index $j$ is greater than the reachable transmitter vehicle ID. ( $j$ - as median vehicle)

- Case - 2: distance between the couple of minimum distance vehicle within the cluster.

- Case - 3: distance between the couple of maximum distance vehicle within the cluster.

Through the above cluster detection mechanism the better cluster formation is identified. Additionally, the clusters are formed with the proposed SRC protocol to broadcast a message in a better VANET environment. 


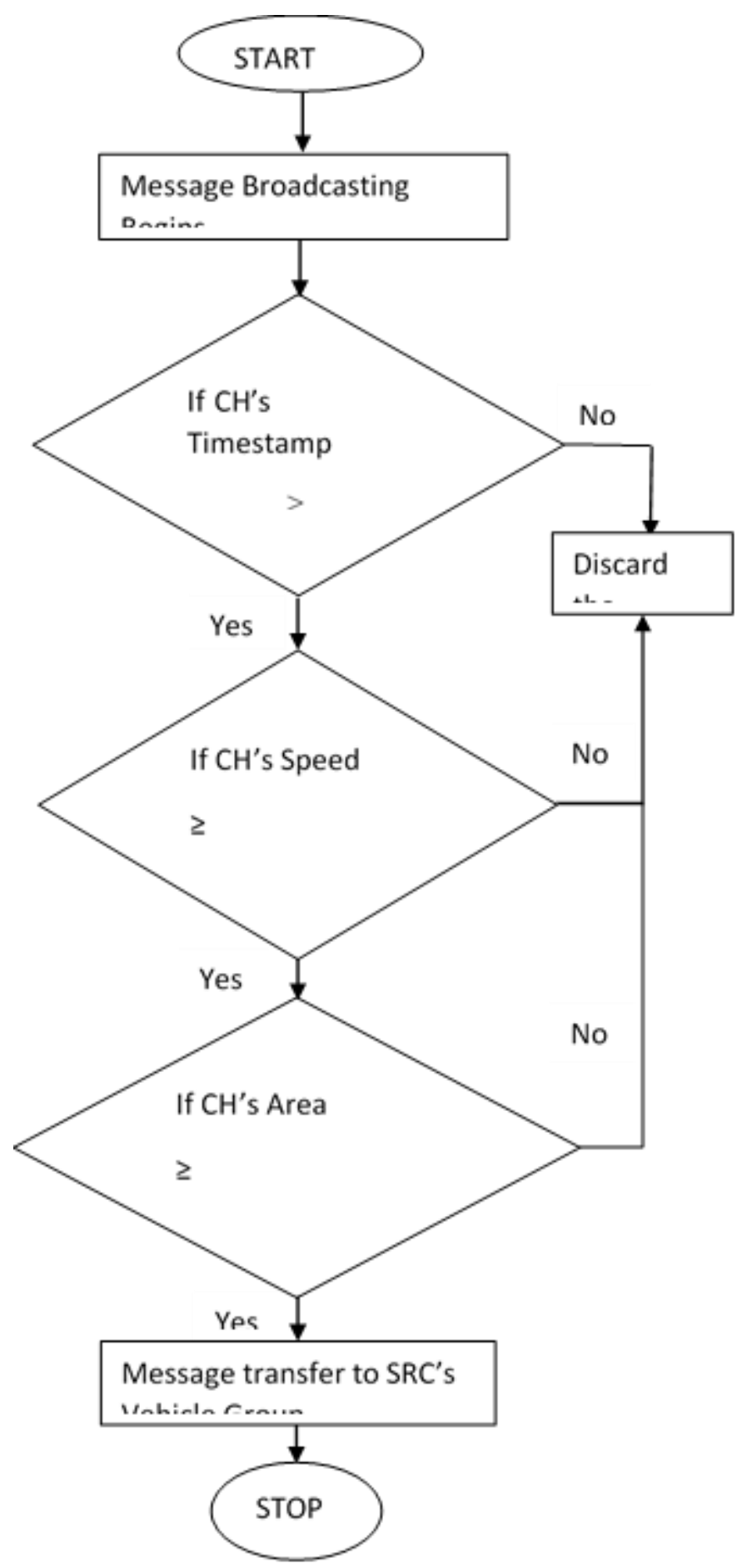

Fig. 4. Flow of SRC Protocol

The Figure 4 presents the flow of SRC protocol. The $\mathrm{CH}$ forwards the data to the $\mathrm{CM}$ as Zone of Interest.

\section{Simulation Results}

This section presents the performance of SRC protocol is implemented using NS2.34. The performance of the proposed protocol is compared with the existing schemes ROAC-B, EWCA and CDP. The various parameters used for the proposed simulation is detailed in Table I. 
TABLE I. SIMULATION PARAMETERS

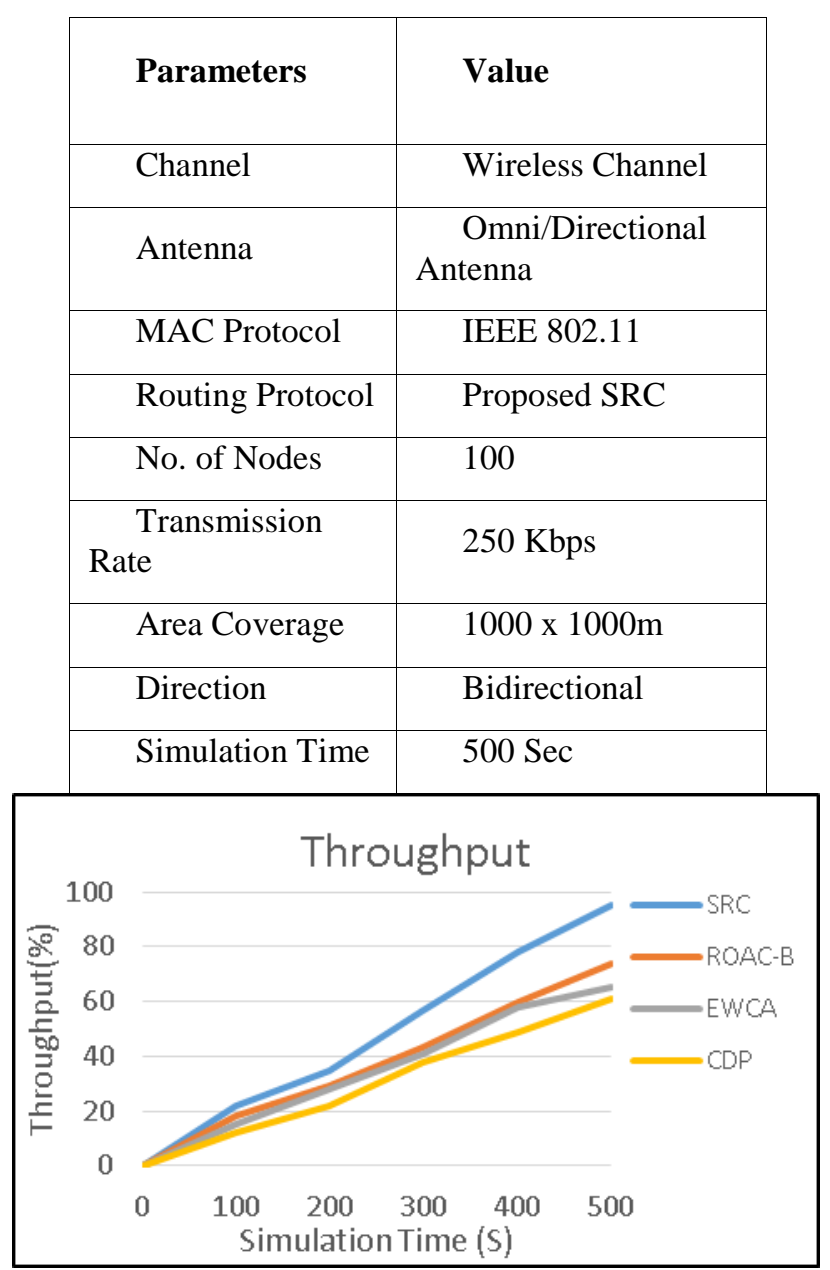

Fig. 5. Throughput

Figure 5 compares the proposed SRC protocol with the existing schemes. SRC achieves $95 \%$ of throughput than ROAC-B (74\%), EWCA (65\%) and CDP (61\%).

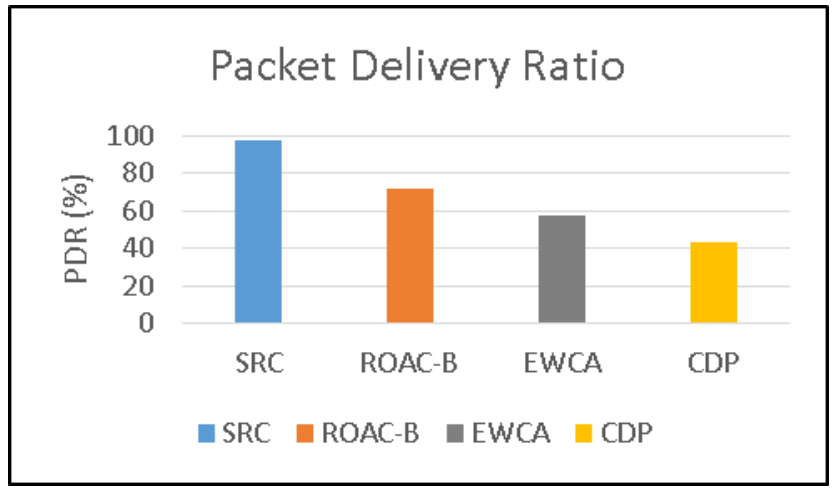

Fig. 6. Packet Delivery Ratio

Figure 6 shows the PDR (\%) between proposed SRC with the other existing schemes. SRC maintains $98 \%$ of PDR whereas ROAC-B maintains 72\%, EWCA retains 58\% and CDP comes with $43 \%$. It shows that the SRC maintains a better PDR than the existing schemes. 


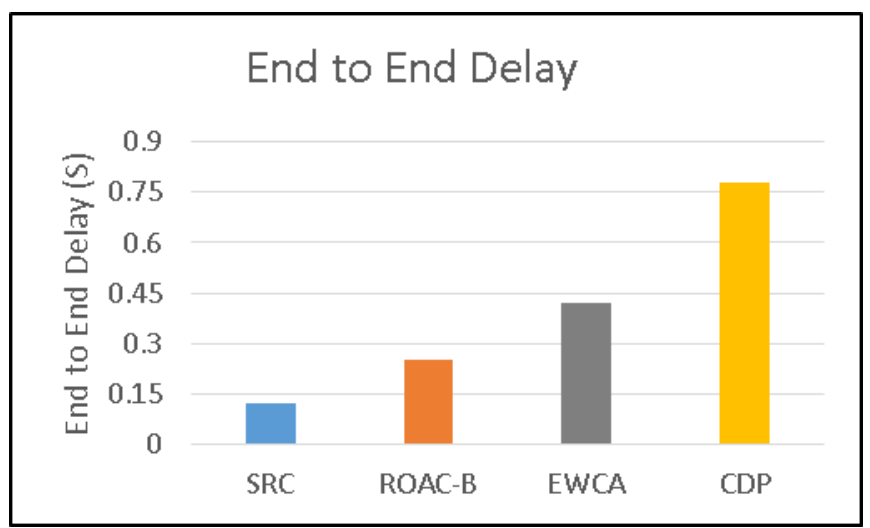

Fig. 7. End to End Delay

The Figure 7 presents End to End Delay between the proposed SRC and the other existing works. The proposed SRC maintains a less delay as $0.12 \mathrm{Sec}$ compared to ROAC-B 0.25 Sec, EWCA with 0.42 Sec and CDP maintains $0.78 \mathrm{Sec}$.

The above figures 5, $6 \& 7$ proves that the proposed SRC maintains a better performance than the abovementioned existing schemes.

\section{Conclusion \& Future Work}

The VANET is an emerging network where the vehicles are transmitting the data about the traffic situation and other relevant information to the nearby vehicles. Even though the VANET is efficient in communicating and transferring the data, the broadcast storm occurs often to detains the performance of the network. To overcome the broadcast storm, there are number of effective protocols are defined. Since, the broadcast storm is not eradicated in the network. Therefore, the proposed SRC protocols found an effective technique to retransmit the data to the Zone of Interest to eradicate the storm and the performance of the SRC proves the broadcast storm is vanished in the VANET. In future, to improve the security in preserving the data transmission can be included with the SRC to implement the same protocol in the Internet of Vehicles (IoV)..

\section{References}

Mchergui, A., Moulahi, T., \& Nasri, S. (2019, June). Relay Selection Based on Deep Learning for Broadcasting in VANET. In 2019 15th International Wireless Communications \& Mobile Computing Conference (IWCMC) (pp. 865-870). IEEE.

Jia, K., Hou, Y., Niu, K., Dong, C., \& He, Z. (2019). The delay-constraint broadcast combined with resource reservation mechanism and field test in VANET. IEEE Access, 7, 59600-59612.

Das, D., \& Misra, R. (2020). EASBVN: efficient approximation scheme for broadcasting in vehicular networks. Wireless Networks, 1-11.

Li, S., Liu, Y., \& Wang, J. (2019). An efficient broadcast scheme for safety-related services in distributed TDMA-based VANETs. IEEE Communications Letters, 23(8), 1432-1436.

Mchergui, A., Moulahi, T., Othman, M. T. B., \& Nasri, S. (2020). Enhancing VANETs Broadcasting Performance with Mobility Prediction for Smart Road. Wireless Personal Communications, 1-13.

Ramalingam, M., \& Thangarajan, R. (2020). Mutated k-means algorithm for dynamic clustering to perform effective and intelligent broadcasting in medical surveillance using selective reliable broadcast protocol in VANET. Computer Communications, 150, 563-568.

Lin, Z., Sun, Y., Tang, Y., \& Liu, Z. (2020). An efficient message broadcasting MAC protocol for VANETs. Wireless Networks, 26(8), 6043-6057.

Mchergui, A., Moulahi, T., \& Nasri, S. (2020). BaaS: Broadcast as a service cross-layer learning-based approach in cloud assisted VANETs. Computer Networks, 182, 107468.

Selvi, M., \& Ramakrishnan, B. (2020). Lion optimization algorithm (LOA)-based reliable emergency message broadcasting system in VANET. Soft Computing, 24(14), 10415-10432.

Naderi, M., Zargari, F., \& Ghanbari, M. (2019). Adaptive beacon broadcast in opportunistic routing for VANETs. Ad Hoc Networks, 86, 119-130.

Srivastava, A., Prakash, A., \& Tripathi, R. (2020). Location based routing protocols in VANET: Issues and existing solutions. Vehicular Communications, 23, 100231.

Hamdi, M. M., Audah, L., Rashid, S. A., Mohammed, A. H., Alani, S., \& Mustafa, A. S. (2020, June). A review of applications, characteristics and challenges in vehicular ad hoc networks (VANETs). In 2020 International Congress on Human-Computer Interaction, Optimization and Robotic Applications (HORA) (pp. 1-7). IEEE. 
Benrhaiem, W., Hafid, A. S., \& Sahu, P. K. (2016, May). Multi-hop reliability for broadcast-based VANET in city environments. In 2016 IEEE International Conference on Communications (ICC) (pp. 1-6). IEEE.

Selvi, M., \& Ramakrishnan, B. (2018, October). An efficient message prioritization and scheduled partitioning technique for emergency message broadcasting in VANET. In 2018 3rd International Conference on Communication and Electronics Systems (ICCES) (pp. 776-781). IEEE.

Pramuanyat, N., Nakorn, K. N., Kawila, K., \& Rojviboonchai, K. (2016, July). LARB: Location-aware reliable broadcasting protocol in VANET. In 2016 13th International Joint Conference on Computer Science and Software Engineering (JCSSE) (pp. 1-6). IEEE.

Oliveira, R., Montez, C., Boukerche, A., \& Wangham, M. S. (2017). Reliable data dissemination protocol for VANET traffic safety applications. Ad Hoc Networks, 63, 30-44.

Ramalingam, M., \& Thangarajan, R. (2017). Weight Value Based Clustering for Dissemination of Emergency Message with Selective Reliable Broadcasting in VANETs. Asian Journal of Research in Social Sciences and Humanities, 7(1), 492-500.

Abbasi, H. I., Voicu, R. C., Copeland, J., \& Chang, Y. (2019). Towards fast and reliable multi-hop routing in VANETs. IEEE Transactions on Mobile Computing.

Sattar, S., Qureshi, H. K., Saleem, M., Mumtaz, S., \& Rodriguez, J. (2018). Reliability and energy-efficiency analysis of safety message broadcast in VANETs. Computer Communications, 119, 118-126 